\title{
Imaging features of adrenal masses
}

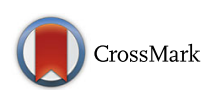

\author{
Domenico Albano ${ }^{1 *} \mathbb{D}$, Francesco Agnello², Federico Midiri², Giusy Pecoraro ${ }^{2}$, Alberto Bruno², Pierpaolo Alongi ${ }^{3}$, \\ Patrizia Toia ${ }^{2}$, Giuseppe Di Buono ${ }^{4}$, Antonino Agrusa4, Luca Maria Sconfienza ${ }^{1,5}$, Salvatore Pardo², \\ Ludovico La Grutta ${ }^{6}$, Massimo Midiri ${ }^{2}$ and Massimo Galia ${ }^{2}$
}

\begin{abstract}
The widespread use of imaging examinations has increased the detection of incidental adrenal lesions, which are mostly benign and non-functioning adenomas. The differentiation of a benign from a malignant adrenal mass can be crucial especially in oncology patients since it would greatly affect treatment and prognosis. In this setting, imaging plays a key role in the detection and characterization of adrenal lesions, with several imaging tools which can be employed by radiologists. A thorough knowledge of the imaging features of adrenal masses is essential to better characterize these lesions, avoiding a misinterpretation of imaging findings, which frequently overlap between benign and malignant conditions, thus helping clinicians and surgeons in the management of patients. The purpose of this paper is to provide an overview of the main imaging features of adrenal masses and tumor-like conditions recalling the strengths and weaknesses of imaging modalities commonly used in adrenal imaging.
\end{abstract}

Keywords: Magnetic resonance imaging, Computed tomography, Adenoma, Adrenal, Chemical shift imaging

\section{Keypoints}

- Adenoma is the most common adrenal tumor

- Washout evaluation of adrenal masses on CECT is the gold standard technique

- Chemical shift MR imaging is a useful tool to identify intra-lesional fat content

- DWI is not useful to differentiate adrenal lesions

- Nuclear medicine techniques are helpful in some specific settings

\section{Introduction}

The adrenal gland is a site of several pathologic conditions, including hyperplasia, hemorrhage, and malignant and benign masses. Tumors can be hyperfunctioning, when producing an increased amount of hormones leading to endocrine disorders, or non-hyperfunctioning, which are characterized by normal hormone levels. Therefore, in some cases, these lesions can be clinically suspected, but in most cases adrenal masses are discovered incidentally. Indeed, the increasing use of imaging investigations, including ultrasound (US), computed

\footnotetext{
* Correspondence: albanodomenico@me.com

'Unità di Radiologia Diagnostica ed Interventistica, IRCCS Istituto Ortopedico

Galeazzi, Via Riccardo Galeazzi 4, 20161 Milan, Italy

Full list of author information is available at the end of the article
}

tomography (CT), and magnetic resonance (MR), has led to an increase in the detection of such incidental lesions [1-4]. Incidental adrenal masses are common, occurring in about $3-7 \%$ of adults [3], with the majority of them being benign non-functioning adenomas [4]. Characterization of an adrenal mass as benign or malignant is critical and imaging plays a key role in influencing the clinical management of patients. In this setting, the role of radiologists is crucial in both the choice of imaging modality and protocol technique to be used and interpretation of imaging findings. US has a limited part in the assessment of adrenals due to its low accuracy in the detection and characterization of small adrenal neoplasms, in comparison to $\mathrm{CT}$ and MR, being useful to differentiate a cystic from a solid mass. Nevertheless, US has still a major role in neonatal patients in which the adrenals are bigger compared to the kidneys. CT represents the first-level imaging modality for the evaluation of adrenal lesions, since it permits a quick execution ensuring high spatial resolution, with findings of pre-contrast images and post-contrast behavior being commonly used to achieve a correct diagnosis [5]. MR still remains a second-level technique, although its advantages include multiparametricity, multiplanarity, higher contrast resolution in comparison with CT, and the added value of chemical shift imaging (CSI) [6]. 
Both CT and MR have therefore a pivotal role in adrenal imaging. When the clinical laboratory picture shows a suspicious functioning adrenal lesion, nuclear medicine techniques such as scintigraphy with 131/ ${ }^{123}$ I-meta-iodo-benzyl-guanidine (MIBG) and Octreotide can be used. Moreover, positron emission tomography (PET) has also shown to be helpful for the evaluation of non-hyperfunctioning adrenal masses with high accuracy in the characterization of malignant lesions [7].

The purpose of this paper is to give an overview of the imaging features of adrenal masses and tumor-like conditions with an emphasis on CT and MR. We will also briefly discuss the recent advancements in diagnostic radiology and nuclear medicine modalities.

\section{Anatomy}

The adrenals are paired retroperitoneal glands that lie superiorly and anteromedially to the kidneys. They are triangular-shaped with the right adrenal gland being usually more pyramidal in shape [8]. The adrenal glands are made of an outer cortex deriving from the mesoderm and an inner medulla deriving from the neural crest. In the cortex, the zona glomerulosa produces mineralocorticoids, the zona fasciculata glucocorticoids, and the zona reticularis sex steroids or gonadocorticoids, whereas the medulla produces catecholamines, adrenaline, and noradrenaline. The adrenals are supplied by three arteries: superior (from the inferior phrenic arteries), middle (from the abdominal aorta), and inferior suprarenal arteries (from the renal arteries). The right adrenal vein ends into the inferior vena cava, while the left adrenal vein ends into the left renal vein or inferior phrenic vein. The lymphatic vessels drain to the para-aortic nodes. The innervation of adrenals comes from the splanchnic nerves arising from the aortic and renal plexuses [9].

\section{Benign adrenal tumors and tumor-like conditions Cortical adenoma}

Adenoma is the most common benign adrenal tumor, with no malignant evolution, arising from the cortex and consisting of clear cells with abundant intracytoplasmatic fat, which is the key element for the differential diagnosis with malignant neoplasms at imaging. Typically, the remaining portion of the adrenal gland and the contralateral adrenal appear atrophic. Large adenomas may show cystic components, calcifications, and hemorrhagic areas [10].

Incidence of adenomas increases with age and most of them are non-functioning, with these lesions being generally asymptomatic and incidentally discovered [11]. Hyperfunctioning adrenal adenomas present with symptoms and signs related to excess hormone secretion, thereby determining Cushing's syndrome (hyperproduction of cortisol) or Conn's syndrome (hyperproduction of aldosterone), while they rarely lead to an adrenal-genital syndrome [12].

The typical adenoma measures less than $3 \mathrm{~cm}$ making challenging its detection through US, in which it appears as a homogeneous and hypoechoic solid lesion with well-defined margins, hypovascularization on Color Doppler examination [13], and hypoenhancing on contrastenhanced US (CEUS) [14]. At unenhanced CT, adenomas are usually well-demarcated round or oval lesions, with homogeneous and relatively low attenuation values (lower than 10 Hounsfield Units [HU]), due to high fat content [15]. However, unenhanced CT alone is not always diagnostic since $15-30 \%$ of adenomas are lipid-poor, thereby showing higher attenuation values. In these cases, additional imaging after intravenous contrast administration is required to differentiate adenoma from non-adenomas. The evaluation of the enhancement pattern of adrenal lesions needs a further late phase after the acquisition of the venous phase. There are different $\mathrm{CT}$ protocols suggested for the evaluation of adrenal masses; however, there is evidence that a 15-min post-contrast protocol has the highest diagnostic accuracy [16-20].

Absolute percentage washout (APW) is calculated using the following formula:

(enhanced $\mathrm{HU}-15$-min delayed $\mathrm{HU}$ )

/ (enhanced HU-unenhanced HU) $\times 100 \%$

Relative percentage washout (RPW) is used when unenhanced $\mathrm{CT}$ value is not available, and the enhanced values are compared with 15 -min delayed scans, by using the following formula:

(enhanced HU-15-min delayed HU) $/($ enhanced HU) $\times 100 \%$

If the APW is $>60 \%$ or RPW is $>40 \%$ after 15 -min from contrast administration, this is indicative of adenoma, with sensitivity and specificity of $88 \%$ and $96 \%$ at the APW and sensitivity and specificity of $83 \%$ and $93 \%$ at the RPW, respectively [5]. This method enables to differentiate adenomas, which enhance quickly and show rapid washout, from non-adenomas such as metastases, which instead demonstrate strong enhancement but prolonged washout. Another useful imaging feature is the size, since lesions greater than $4 \mathrm{~cm}$ lay for malignant tumors, and the dimensional growth, considering that adenomas grow slower than malignant masses [21].

MR has similar diagnostic accuracy to CT allowing characterizing adenomas regardless of their $\mathrm{CT}$ enhancement (Fig. 1) [6]. On T2-weighted MR images, adrenal adenomas are homogeneous and present intermediate-low signal intensity compared to skeletal muscle or liver; intra-lesional hemorrhage can occur in large adenomas resulting in hyperintense areas on T1-weighted images [20]. An important component of adrenal MR protocol is 

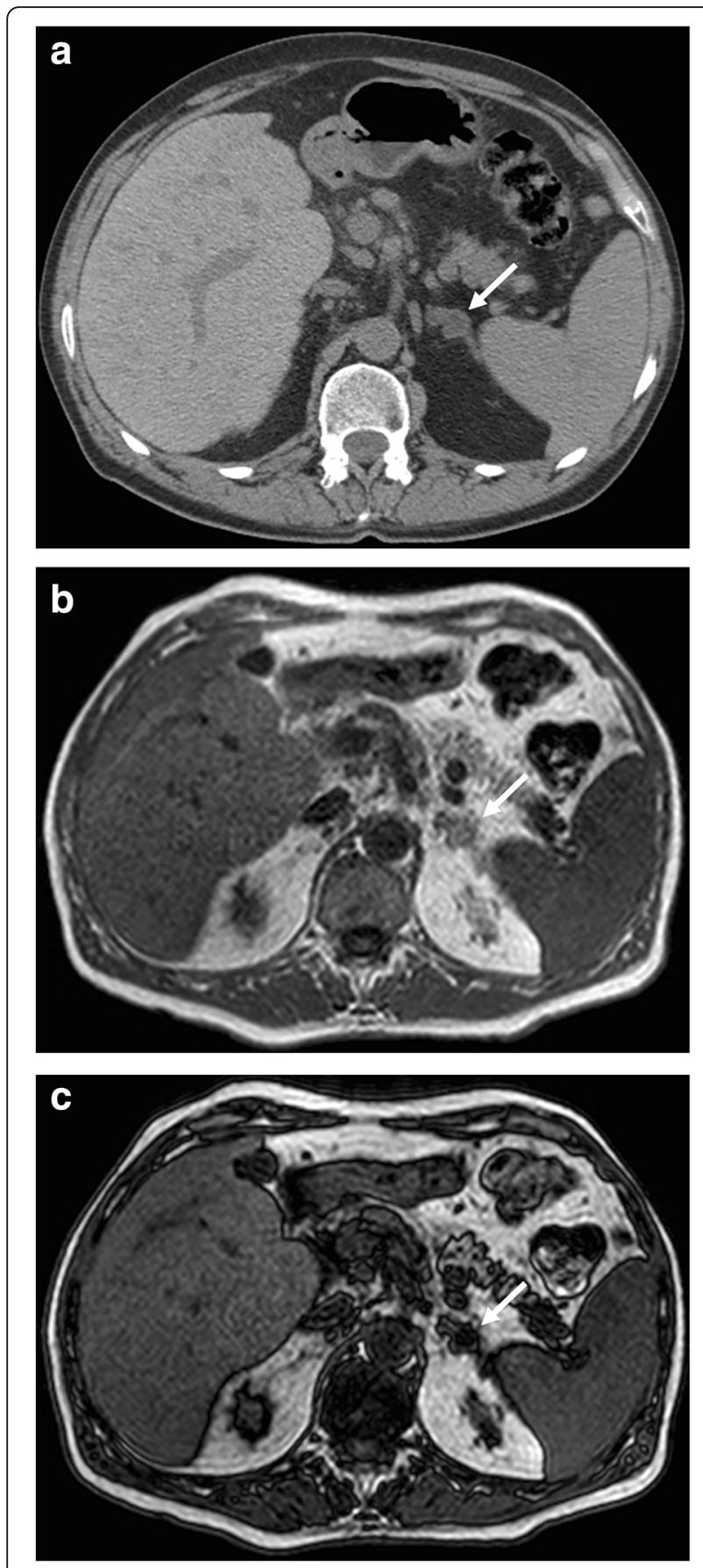

Fig. 1 Adrenal adenoma of a 45-year-old woman. Unenhanced CT (a) shows a left adrenal mass (arrow) with lower attenuation values than $10 \mathrm{HU}$. Axial T1-weighted in-phase (b) and out-of-phase (c) gradient recalled echo MR images show prominent loss of signal on out-of-phase

CSI. This modality uses the different precession frequencies of protons in both water and fat within the same voxel and creates in-phase and opposed-phase images, which enable to detect intra-lesional fat resulting in a loss of signal intensity in the opposed phased images [6]. Thus, most adrenal adenomas demonstrate a loss of signal intensity on out-of-phase images, and a decrease in signal intensity of more than $20 \%$ is considered diagnostic of adenoma. The MR sensitivity for adenomas measuring $10-20 \mathrm{HU}$ is nearly $100 \%$, while that for lipid-poor adenomas measuring greater than $30 \mathrm{HU}$ is significantly lower $(13-75 \%)[10,22]$. The loss of signal intensity can be also quantified using parameters such as the spleno-adrenal ratio (ASR) and the signal intensity index (SII). An ASR $<70 \%$ is highly specific for adenoma and has sensitivity of 78\%; while using SII, a loss of signal intensity greater than $5 \%$ has allowed to identify adenomas in $100 \%$ of cases [23]. Of note, Namimoto et al. reported similar results in the identification of adenomas by comparing three-point Dixon techniques for CSI and conventional dual-echo imaging [24].

In comparison to contrast-enhanced CT (CECT), contrast-enhanced MRI with gadolinium washout studies does not appear to exhibit the same diagnostic strength. Thus, CECT remains the gold standard technique, especially in the evaluation of lipid-poor adenomas. Data related to diffusion-weighted imaging (DWI) of adrenal masses have been disappointing mainly due to benign adrenal adenomas' propensity to demonstrate restricted pattern of diffusion. Miller et al. retrospectively evaluated 160 adrenal lesions and found that apparent diffusion coefficient (ADC) was not useful to differentiate benign from malignant adrenal neoplasms [25]. Sandrasegaran et al. also found that ADC values were not helpful for this purpose [26].

Over the last years, CT perfusion imaging has been widely used for the diagnosis and characterization of tumors, but few studies about its potential role in the characterization of adrenal lesions have been reported. Perfusion imaging evaluates tissue vascularity after contrast media administration by measuring changes in tissue enhancement and perfusion. Previous studies have showed that a perfusion parameter indicated as blood volume is significantly higher in adenomas, being potentially helpful to differentiate adenomas from non-adenomas [27].

Regarding the role of dual energy CT, studies have reported that this imaging modality allows an accurate characterization of lipid-rich adenoma evaluating that a decrease in attenuation of an adrenal lesion between 140 and $80 \mathrm{kVp}$ is a highly specific sign of adenoma. However, because an increase in attenuation at $80 \mathrm{kVp}$ is seen on metastatic lesions and some adenomas, the sensitivity of this modality is still low [28].

Few data have been published on nodule characterization by histogram analysis, thus there is poor knowledge regarding the potential application of this tool. This method also takes advantage of the higher intracytoplasmatic fat content of adenomas. With a threshold value of $>10 \%$ negative pixels, it is possible to 
identify many benign adrenal nodules with attenuation values $>10 \mathrm{HU}$ on unenhanced $\mathrm{CT}$ with high accuracy; moreover, a threshold of $>10 \%$ negative pixels seems to guarantee $100 \%$ specificity [29]. New interesting perspectives include the characterization of adrenal lesions by using texture analysis, with some reports already showing promising results by some texture analysis-derived features [30].

\section{Hyperplasia}

Hyperplasia refers to nonmalignant diffuse or focal enlargement of the adrenal gland, which is an uncommon cause of ACTH-independent Cushing's syndrome or Conn's syndrome. Adrenal hyperplasia can be congenital or acquired and is classified according to morphology in diffuse or nodular adrenal hyperplasia [31]. Among the causes of ACTH-independent Cushing's syndrome, we should recall primary pigmented nodular adrenal disease (PPNAD), which is a benign condition generally characterized by mild symptoms related to hypercortisolism. In patients with PPNAD, imaging allows to identify multiple small nodules in normal-sized or enlarged adrenal glands [32].
At CT, diffuse hyperplasia is characterized by the increased volume of both adrenals presenting smooth margins, a homogeneous structure, and often some nodules of variable size, usually smaller than $5-10 \mathrm{~mm}$, but even up to $2 \mathrm{~cm}$. A large nodule is rarely detected and it differs from the adenoma because the former lies within enlarged glands, while the latter appears within an atrophic adrenal gland or associated with atrophy of the contralateral gland [33]. Hyperplasia contains variably sized groups of lipid-rich cells, thereby showing a significant overlap with adenoma in terms of lesion attenuation and APW or RPW [33]. On MR, hyperplasia shows signal intensity similar to that of the normal gland but appears as homogeneous or nodular enlarged gland; this appearance helps in the differentiation from other nodular pathologies, which are generally hyperintense on T2-weighted images (Fig. 2) [34].

\section{Adrenal infection}

Tuberculosis is one the most frequent adrenal infections, still being the primary cause of primary adrenal insufficiency in developing countries. Generally, tuberculosis affects both adrenal glands and the clinical picture is that of adrenal insufficiency, including weakness, nausea,
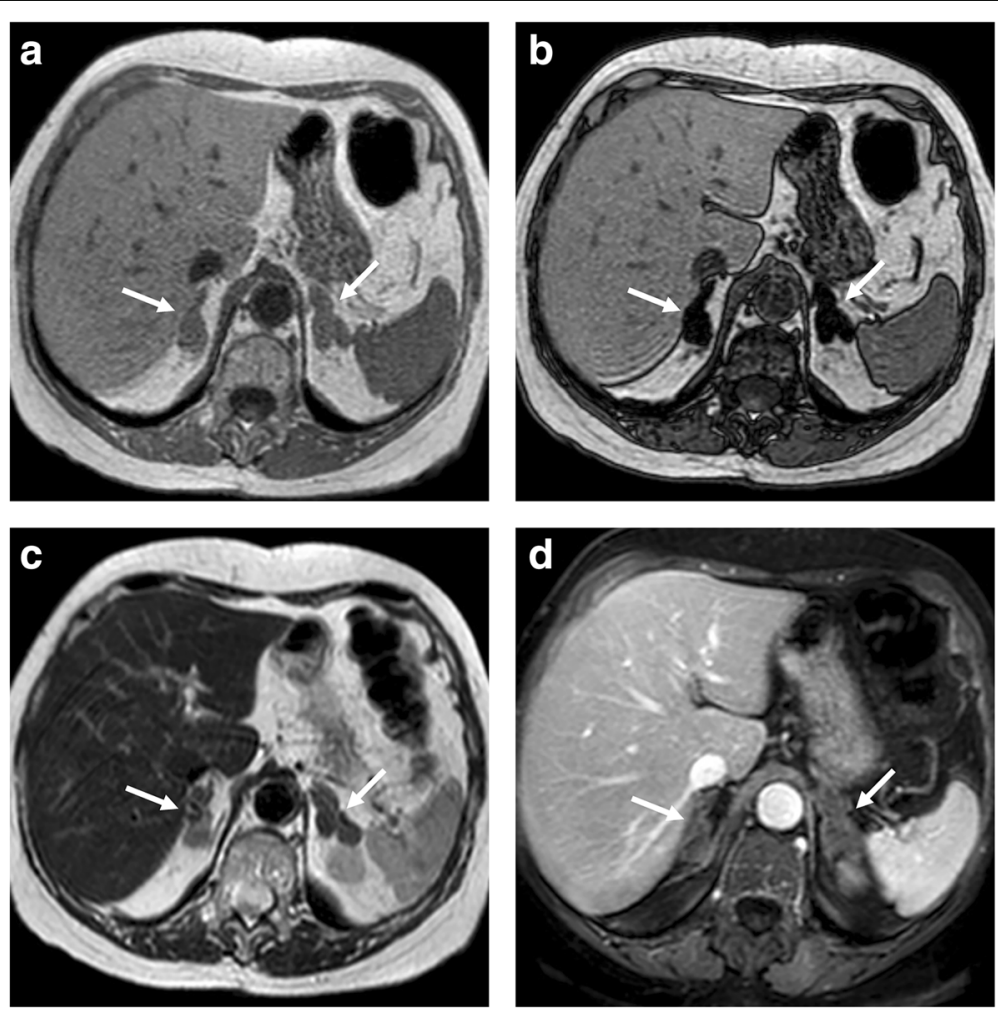

Fig. 2 Bilateral adrenal hyperplasia in a 75-year-old woman. Axial T1-weighted in-phase (a) and out-of-phase gradient recalled echo (c), T2weighted fast spin echo (c), and three dimensional fat-suppressed T1-weighted gradient recalled echo image in portal phase after contrast injection (d) show the nodular enlargement of both adrenal glands (arrows) with loss of signal on out-of-phase (b) 
anorexia, weight loss, abdominal pain, and hypotension. Depending on the disease stage, CT and MR show different findings. At first, a mass-like enlargement with preserved contour is commonly detected. Central low attenuation on unenhanced CT and peripheral enhancement on post-contrast $\mathrm{CT}$ and MR images is another typical imaging finding due to a central caseous necrosis area [35]. Later, a decrease in size of adrenal glands is observed with the frequent occurrence of diffuse, localized, or punctuated calcifications, with atrophic adrenal glands with calcifications being considered highly suspicious for chronic tuberculosis [36]. During antituberculosis therapy, the caseous necrosis can be replaced by fibrous tissue and cicatrix, thereby leading to a more homogeneous enhancement or central calcific deposits [36]. Another adrenal infection mimicking tuberculosis is histoplasmosis, although neoplastic conditions such as metastases or lymphoma may show similar imaging features. Thus, histologic evaluation of adrenal tissue after biopsy is generally required to achieve the correct diagnosis.

\section{Adrenal hemorrhage}

Adrenal hemorrhage is a condition with different etiopathogenetic, clinical, and prognostic aspects depending on whether it occurs in children or in adults. Adrenal hemorrhage can result from trauma, anticoagulant therapy, complicated pregnancy, sepsis, or stress, such as surgery [37, 38]. Moreover, adrenal hemorrhage may occur within adrenal lesions such as adenoma, myelolipoma, pheochromocytoma, metastasis, and cortical carcinoma. Trauma is the most common cause, with post-traumatic hemorrhage being usually unilateral [37]. Bilateral hemorrhage occurs in $20 \%$ of cases, and adrenal insufficiency secondary to hemorrhage is extremely rare [38]. US represents the first-level imaging modality in children. Acute hemorrhage appears as a mass with a diameter of $3-4 \mathrm{~cm}$ with a mixed echostructure, predominantly hyperechogenic due to blood clots within the hematoma. In the subsequent phases, the mass progressively becomes liquid and then hypo-anechoic due to the lysis of the clots. At Color Doppler, adrenal hemorrhage shows no vascularization [38].

On CT, acute hemorrhage is characterized by high attenuation values, in post-traumatic cases, associated with hemorrhagic suffusion of periadrenal fat and retroperitoneal bleeding (Fig. 3). A chronic hematoma appears as a mass with a hypoattenuating center with or without calcifications, also termed adrenal pseudocyst. Hematomas usually decrease in size and may spontaneously disappear. Calcifications may develop in the late stage of hemorrhages [38].

On MR, signal intensity of adrenal hemorrhage depends on its stage. In the acute stage (first 7 days), the hematoma
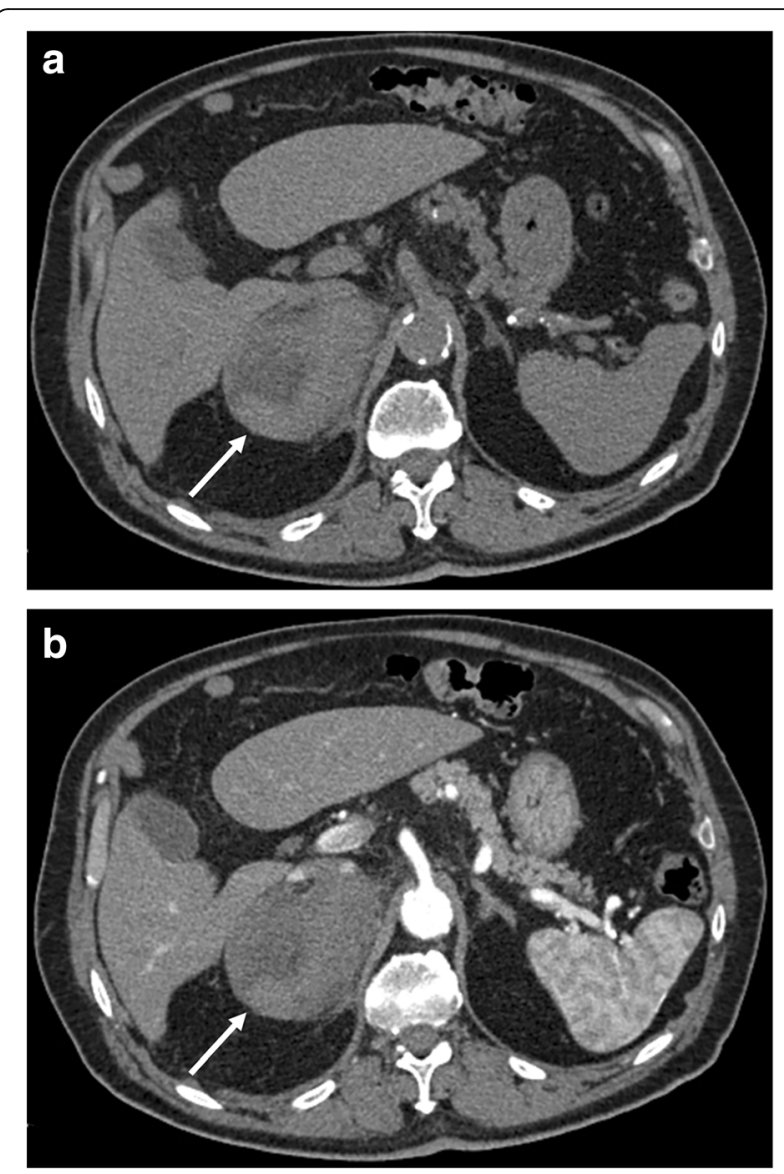

Fig. 3 Right adrenal hemorrhage in a 61-year-old man with acute abdominal pain after starting anticoagulant therapy. Axial unenhanced (a) and arterial (b) phase CT images show a large right adrenal hematoma (arrow) with relatively high attenuation values and without enhancement after contrast injection

appears isointense or slightly hypointense on T1-weighted and hypointense on T2-weighted images, due to the presence of deoxyhemoglobin. In the subacute phase (1 week to 7 weeks), the hemorrhage appears hyperintense on both T1- and T2-weighted images due to the paramagnetic effect of the methemoglobin. In the chronic stage (more than 7 weeks), a hypointense rim on T1- and T2-weighted images due to hemosiderin deposition and fibrosis is detectable [38].

\section{Cyst}

Adrenal cysts are uncommon. Traditionally, adrenal cysts have been classified as pseudocysts (resulting from previous hemorrhage), endothelial cysts (with a thin endothelial wall and lactescent liquid, deriving from lymphangiectasia or arteriovenous malformations), epithelial cysts (with thin epithelial wall and serous liquid content), and parasitic cysts (hydatid disease). These cysts can range in size from 1 to $20 \mathrm{~cm}[39,40]$. At US, 
endothelial and epithelial cysts appear as well-circumscribed hypoechoic or anechoic lesions with thin walls, while pseudocysts may have thicker walls. The scattered echogenicity, the presence of debris, or fluid-fluid levels suggest previous hemorrhage into a pseudocyst [40]. On CT, adrenal cysts appear as well-demarcated, non-enhancing, hypoattenuating lesion with water attenuation $(<20 \mathrm{HU})$ and thin walls. Nevertheless, pseudocysts may have a minimally thick cyst wall and complicated fluid [31]. In some cases, internal hemorrhage or calcifications can be detected, especially in pseudocysts and parasitic cysts. On MR, cysts are usually hyperintense on T2-weighted images (Fig. 4). Septations, blood products, or a soft-tissue component and calcifications may be observed in pseudocysts [31].

\section{Myelolipoma}

Adrenal lipomatous tumors are neoplasms with a significant proportion of adipose tissue, with myelolipoma being the most common type. Myelolipoma is a benign tumor, usually unilateral, small, and often incidentally discovered. It consists of mature fat and hematopoietic cells from myeloid, erythroid, and megakaryocytic lines. The amount of fatty component is variable with some tumors showing small regions of fat within a predominantly soft-tissue mass, whereas others consist almost completely of fat. It can infrequently contain calcifications or show partial replacement by hemorrhage or fibrosis. Large lesions tend to become symptomatic due to pressure effects and to intra-lesional hemorrhage or infarctions. While smaller lesions can be managed conservatively, those with a size of more than $4 \mathrm{~cm}$ are likely to be surgically removed. Indeed, the American Association of Clinical Endocrinologists and American Association of Endocrine Surgery (AACE/AAES) 2009 guidelines recommend adrenalectomy for all myelolipomas measuring more than $4 \mathrm{~cm}$ [41].

At US, fat-predominant lesions appear hyperechoic, while myelolipomas mainly composed of myeloid elements appear hypoechoic. Frequently, the lesion has mixed hyperechoic and hypoechoic areas, due to varying amounts of fat and myeloid elements [42].

At CT, myelolipoma appears as a round mass with well-defined borders. The density varies depending on the proportion of fat and myeloid components. Internal inhomogeneity is related to intra-lesional septa, necrosis, hemorrhage, or calcifications. Thus, myelolipoma goes from totally fat lesions with thin circles of parenchymatous tissue to clearly parenchymatous forms with small areas of fat. At CT, myelolipoma is easily diagnosed because gross fat, with attenuation values $<-30 \mathrm{HU}$, is almost always detectable. After contrast agent injection, myelolipoma typically shows slight enhancement because it is poorly vascularized $[31,42]$.
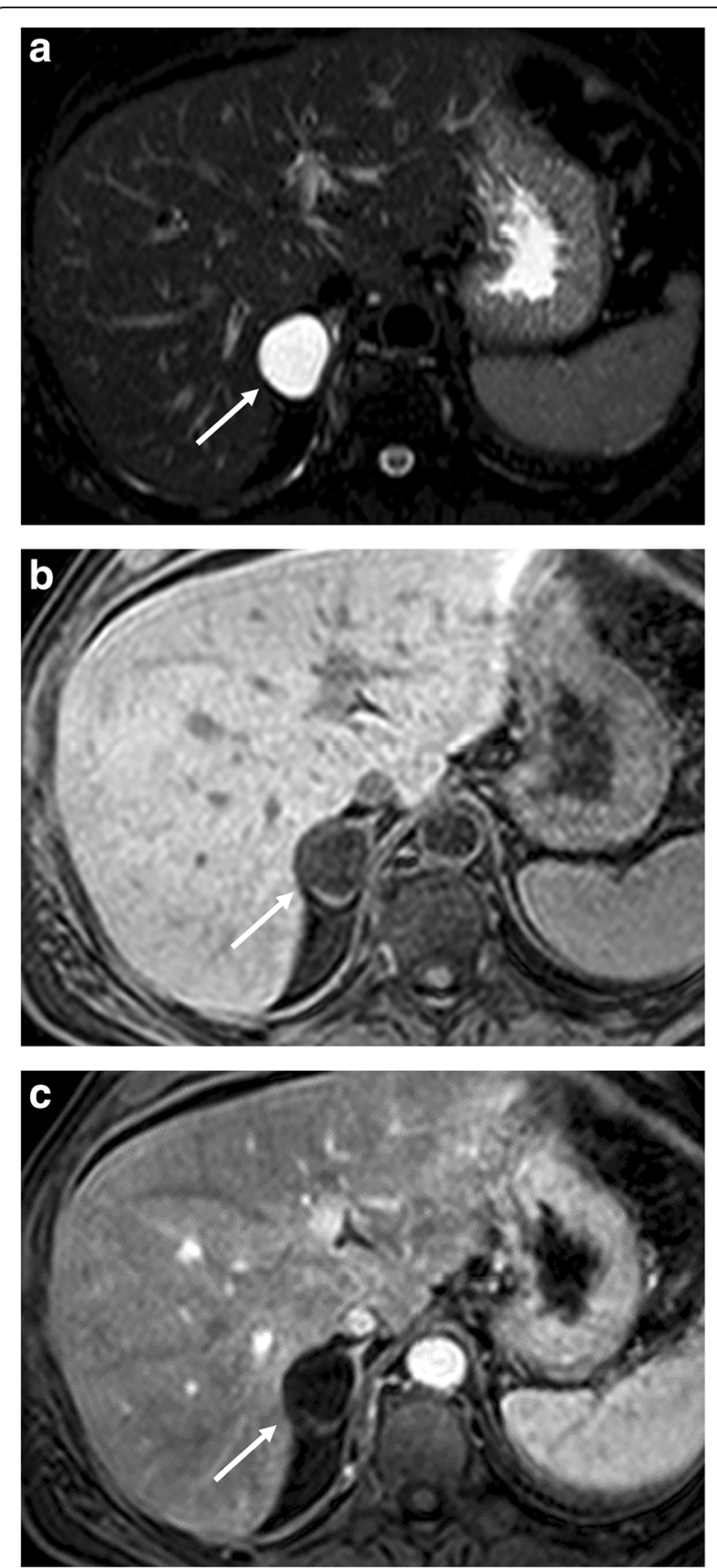

Fig. 4 Right adrenal cyst in a 50-year-old woman. Axial T2-weighted fat-saturated fast spin echo MR image (a), three-dimensional fatsuppressed T1-weighted gradient recalled echo images before (b) and after paramagnetic contrast administration (c) show a right adrenal cystic lesion (arrow) with low T1 signal intensity, high T2 signal intensity, no contrast enhancement and no septations, blood products, soft-tissue components, or calcifications

On MR, the fat macroscopic components are hyperintense on T1- and T2-weighted images, while the hematopoietic parts are hypointense on T1- and moderately hyperintense on T2-weighted images. The diagnosis becomes certain when the hyperintensity on 
T1-weighted images disappears on sequences with fat suppression [41, 42]. Similar to renal angiomyolipomas, the presence of the India ink artifact on CSI at the myelolipoma-adrenal interface or within an adrenal mass on opposed-phase images should indicate a myelolipoma [43]. Fat-suppressed images show greater loss of signal intensity than CSI sequences, as the presence of macroscopic adipose tissue is typical of myelolipoma (Fig. 5). On contrast-enhanced MR, myelolipoma may have enhancement of the myeloid component. Based on MR characteristics, three different types of myelolipoma were described. The first is mostly composed of fat and homogeneous and hyperintense on T1-weighted images. The second consisted of similar content of fat and myeloid components with a heterogeneous appearance. The third type is mainly composed of myeloid tissue with enhancement after contrast injection [9].

\section{Lipoma}

Adrenal lipomas are rare tumors of mesenchymal origin containing mature fatty tissue and surrounded by a fibrous capsule, similarly to lipomas elsewhere in the body, with only a few cases having been reported in the adrenals [44, 45]. These are well-demarcated lesions composed of lobules of fat tissue. On CT, in contrast to myelolipomas, they show no or minimal soft-tissue attenuation and can contain focal areas of intra-lesional calcifications due to degenerative changes [44, 45].

\section{Pheochromocytoma}

Pheochromocytoma arises from the chromaffin cells of the adrenal medulla and typically produces both norepinephrine and epinephrine [46]. Extra-adrenal pheochromocytomas are uncommon and are termed paragangliomas, originating in the sympathetic ganglia
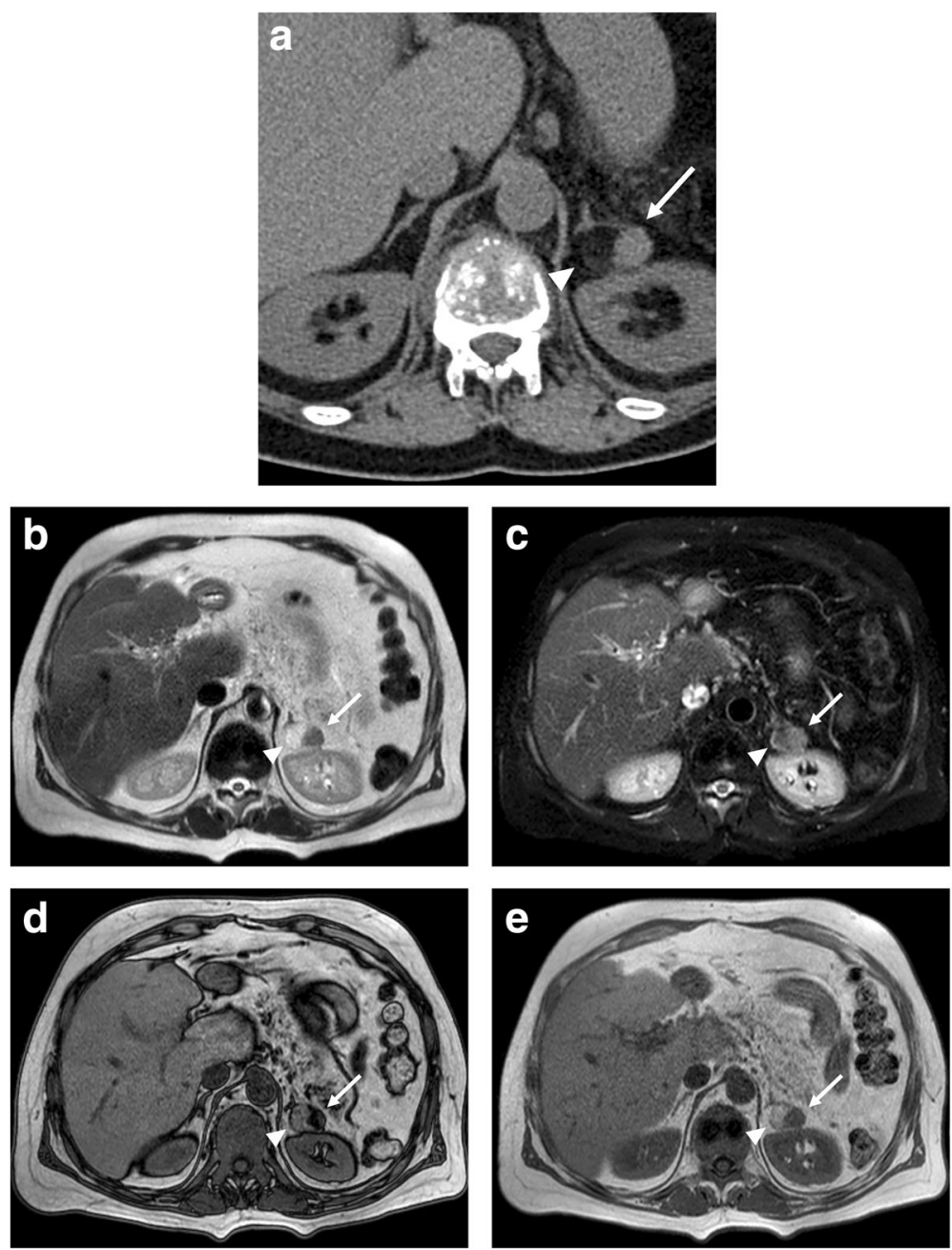

Fig. 5 Left adrenal myelolipoma incidentally discovered in a lumbar CT scan on a 51-year-old man. Unenhanced CT (a) shows a left adrenal mass with macroscopic fat (arrowhead) and a soft-tissue myeloid component (arrow). Axial T2-weighted fast spin echo (b), T2-weighted fat-saturated spin echo (c), T1-weighted in-phase (d), and out-of-phase gradient recalled echo images (e) show the signal drop of macroscopic fat in fatsuppressed T2-weigthed images (c, arrowhead) and show loss of signal on out-of-phase imaging of the myeloid element (e, arrow) 
at the level of the mediastinum, abdomen, and pelvis. Adrenal pheochromocytoma is commonly benign, although $10 \%$ of these lesions can be malignant [46]. Malignant pheochromocytomas are recognized for local infiltration or metastases, usually involving the bone, liver, lymph nodes, lungs, and brain. Clinical symptoms are associated with catecholamine excess (headache, sweating, palpitations, pallor, weight loss, and/or anxiety). This tumor is generally sporadic, although it can be associated with hereditary syndromes including multiple endocrine neoplasia (MEN)-2A, MEN-2B, von Hippel-Lindau, and NF-1. Pheochromocytomas are variable in size ranging from 1.2 to $15 \mathrm{~cm}$ with a mean size of $5.5 \mathrm{~cm}[13,31]$. When a pheochromocytoma is suspected, the first diagnostic step is the evaluation of catecholamines and metanephrines in urine.

The diagnosis of pheochromocytoma at imaging is often challenging due to its complex and variable appearance, related to necrosis, fibrosis, cystic and fatty degeneration, and calcification, which has led to describe it as "imaging chameleons" mimicking other lesions [47]. At US, pheochromocytomas are heterogeneous and well-encapsulated, with hypervascularization at color Doppler and an early arterial pattern of enhancement at CEUS $[48,49]$.

At CT, pheochromocytomas may present solid, cystic, calcific, and/or necrotic components. Smaller tumors tend to display a more uniform attenuation, with a density of 40-50 UH [47, 49]. After contrast administration, pheochromocytomas enhance avidly with some of them showing higher enhancement on the portal venous phase and other on the arterial phase; nevertheless, their APW and RPW are similar to those of adenomas [49]. Therefore, pheochromocytomas often cannot be reliably differentiated from adenomas using CT washout protocols. When lesions are quite large $(>6 \mathrm{~cm})$, intra-lesional hemorrhage, necrosis, or calcifications can be observed, determining the inhomogenous contrast enhancement.

MR is highly sensitive for the diagnosis of pheochromocytomas with a sensitivity of about 98\% [49]. Pheochromocytoma is slightly hypointense on T1-weighted and hyperintense on T2-weighted images [50]. It does not present loss of signal intensity on opposed-phase images, unlike the typical adenoma (Fig. 6). In some cases, areas of fatty degeneration can be encountered, leading to slight signal drop on CSI [6]. Although DWI has not a key role in the differentiation of benign and malignant adrenal lesions $[25,26]$, it is potentially useful to detect pathologic lymph nodes and liver metastases. Nevertheless, a study performed at $3 \mathrm{~T}$ has reported significantly lower ADC values of malignant pheochromocytomas in comparison to benign ones [51]. MR spectroscopy has also been used to characterize adrenal masses and particularly pheochromocytomas, postulating that metabolic content can be measured using that modality. Specifically, it was found that pheochromocytomas exhibit a unique spectral appearance, with the most prominent resonating peak at $6.8 \mathrm{ppm}$, with other resonance peaks at $2.7,3.16$, and $3.8 \mathrm{ppm}$, attributable to catecholamines and catecholamine-derived metabolites. However, the use of this technique has been remarkably limited by the anatomical location of the adrenal glands, as their vicinity to the diaphragm makes the analysis quite demanding [52]. ${ }^{131 / 123}$ I-MIBG scintigraphy is currently the functional nuclear medicine imaging of choice for pheochromocytoma [11] but suffers from drawbacks like limited spatial resolution, difficulty in detection of small tumors $(<1.5-2.0 \mathrm{~cm})$ or large tumors with extensive necrosis/ hemorrhage, lack of tracer uptake in some tumors, and interference with certain medications, leading to false-negative results [53]. Expression of somatostatin receptors by pheochromocytoma facilitates targeted PET imaging with ${ }^{68} \mathrm{Ga}$-DOTA-peptides. In a study of Sharma et al., ${ }^{68} \mathrm{Ga}$-DOTANOC PET/CT showed high diagnostic accuracy (on patient-based analysis sensitivity, specificity, and accuracy of $92 \%, 85 \%$, and $90 \%$, respectively) in patients with a suspicion of pheochromocytoma, and was superior to MIBG imaging [54]. Furthermore, the properties of uptake and storage of catecholamines in pheochromocytomas open the potential use of ${ }^{18} \mathrm{~F}$-FDOPA PET/CT for the evaluation of the relationship between tumor secretion and its biochemical phenotype [55].

\section{Hemangioma}

Hemangioma is an uncommon incidental adrenal lesion generally discovered by CT or MR, especially in women between 40 and 70 years of age. Regarding the histologic types, the most common are capillary and cavernous hemangiomas [56]. Its radiologic features are similar to those of hepatic and soft-tissue hemangiomas. On US, hemangioma has no specific characteristics, appearing as a mass with variable size and echotexture. On CT, small hemangiomas can be homogeneous, whereas larger lesions present calcifications which can be either phleboliths or dystrophic calcifications due to previous hemorrhages [31]. On CECT, hemangioma shows heterogeneous and mainly peripheral enhancement, with or without central filling on delayed phases (Fig. 7) [31]. At MR, hemangioma is hypointense on T1-weighted with a remarkable hyperintensity on $\mathrm{T} 2$-weighted images (lightbulb sign). Hypointense foci can be encountered on $\mathrm{T} 1$ - and $\mathrm{T} 2$-weighted images related to intralesional calcifications. Also on contrast-enhanced MR, hemangioma usually displays peripheral nodular enhancement. 

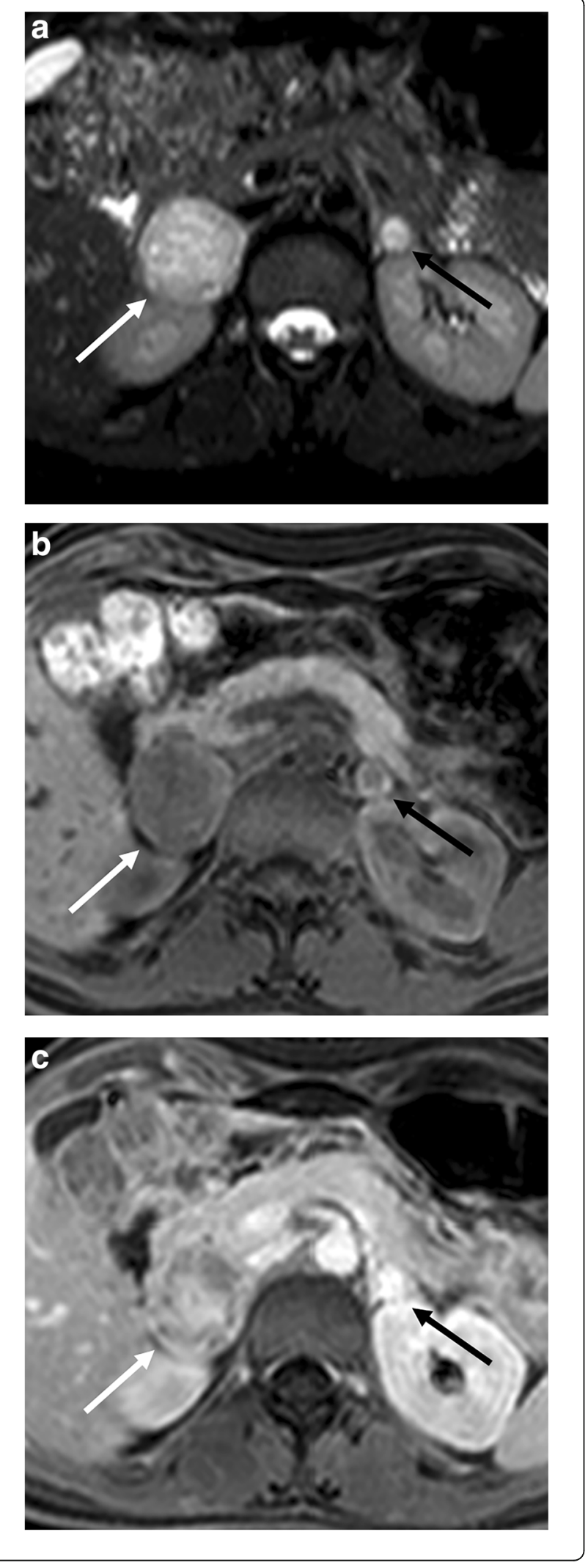

Fig. 6 Bilateral adrenal pheochromocytoma in a 27-year-old woman with multiple endocrine neoplasia type 2. Axial T2-weighted fatsaturated spin echo (a), three-dimensional fat-suppressed T1weighted gradient recalled echo images before contrast injection (b), and in portal phase (c) images show a bilateral adrenal mass with high $\mathrm{T} 2$ signal intensity and strong and heterogenous contrast enhancement. The signal intensity is typically more inhomogenous in larger lesions (white arrow, right adrenal gland) than in smaller ones (black arrow, left adrenal gland)

\section{Lymphangioma}

Lymphangiomas are rare adrenal incidentally discovered benign lesions, being totally asymptomatic and occurring at any age [57]. On CT, lymphangioma appears as a well-encapsulated hypodense lesion $(8-20 \mathrm{HU})$ with an average size of $3 \mathrm{~cm}$ with intra-lesional septations showing contrast enhancement. Lymphangioma can also contain calcifications which appear scattered punctate or thick curvilinear [58]. At MR, lymphangioma does not display specific imaging features showing low signal intensity on T1- and high signal on T2-weighted images.

\section{Schwannoma}

Schwannoma is a rare and usually benign tumor arising from the sheath of the nerves, with only few cases of adrenal schwannomas having been previously described [59]. This lesion is commonly asymptomatic, but in some cases it may present with abdominal pain, generally due to hemorrhage. At $\mathrm{CT}$, it appears as hypodense with inhomogeneous contrast enhancement. Larger lesions usually present degenerative changes including hemorrhagic components, calcifications, and cystic areas, making MR features non-specific, although adrenal schwannoma generally appears isointense on T1-weighted images showing slight hyperintensity on $\mathrm{T} 2$-weighted images $[59,60]$.

\section{Ganglioneuroma}

Ganglioneuroma is a benign tumor arising from the sympathetic nerves or within the adrenals. This lesion is more frequently detected in the mediastinum or retroperitoneum than in the adrenal medulla [61]. Usually, it is a small round mass $(2-3 \mathrm{~cm})$ with well-defined smooth margins and an inhomogeneous appearance due to mixoid components. On CT, it appears as a well-circumscribed solid iso- or hypoattenuating lesion, which may display calcifications, necrosis, and hemorrhagic areas [62]. It remains hypoattenuating on early post-contrastographic phases, while becoming hyperattenuating on delayed phases due to persistent enhancement [62]. On MR, ganglioneuroma is hypointense on T1-weighted and hyperintense on T2-weighted 

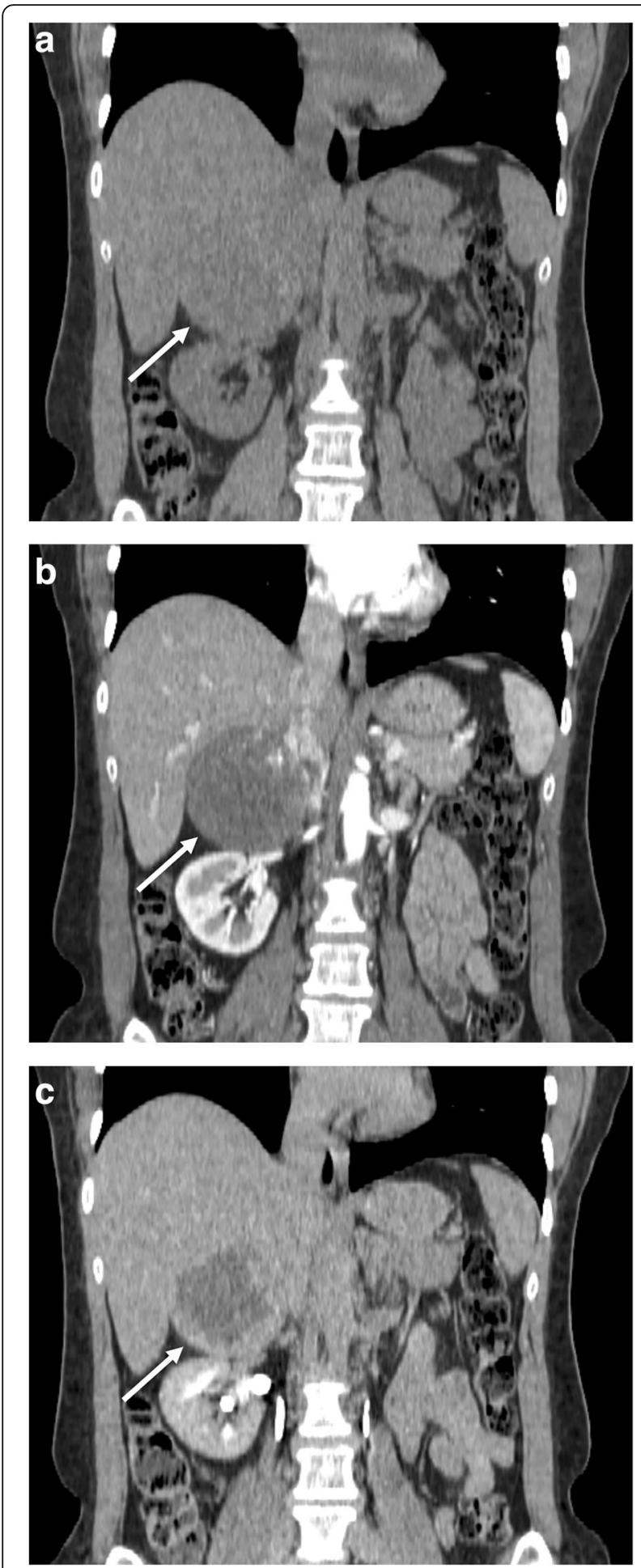

Fig. 7 Right adrenal hemangioma in a 49-year-old woman. Coronal reformatted $C T$ images before (a) and after contrast injection in arterial (b) and late phase (c) show a well-encapsulated large right adrenal lesion (arrow) showing peripheral contrast enhancement with progressive centripetal filling and a hypodense center images, with slow and persistent enhancement on post-contrastographic images [62].

\section{Adenomatoid tumor}

Adenomatoid tumor is an uncommon benign lesion with mesothelial origin, which is generally asymptomatic. On CECT, this tumor appears as a hypoattenuating lesion with heterogeneous contrast enhancement. Calcifications and septations may be observed within the mass. MR findings are non-specific with a variable appearance on T1-weighted, T2-weighted, and post-contrast images [31].

\section{Oncocytoma}

Oncocytoma is a rare epithelial tumor affecting more frequently the left adrenal and showing a mean size of about $9 \mathrm{~cm}$. This neoplasm is generally benign and non-functioning, although few cases of functioning lesions secreting cortisol and androgens have been previously reported [63]. According to the Lin-WeissBisceglia criteria, adrenal oncocytomas can be benign, borderline malignant potential, and malignant lesions [64]. Few malignant metastatic oncocytomas have been described [65]. On CT and MR scans, the lesion appears as a well-demarcated mass with heterogeneous contrast enhancement due to degenerative changes [65]. Fibrous encapsulation is a typical feature of oncocytoma, having been observed in both benign and malignant variants [65]. Unfortunately, the differentiation from cortical carcinoma is still challenging, similar to that observed for renal oncocytomas and clear cell renal carcinoma $[49,66]$.

\section{Malignant adrenal tumors Cortical carcinoma}

Cortical carcinoma is an uncommon malignant adrenal tumor, with a bimodal peak of incidence in childhood and middle age [66]. For unclear reasons, it may be functioning especially in females, who also show more frequently an association with endocrine syndromes including Li-Fraumeni syndrome, Beckwith-Wiedemann syndrome, Carney complex, congenital adrenal hyperplasia, and MEN-1 [66, 67]. Non-functioning lesions are asymptomatic as long as they become so large to determine compression and dislocation of adjacent structures.

At US, this lesion presents as a large mass, generally larger than $6 \mathrm{~cm}$ in size. Small lesions may show homogeneous echotexture, whereas larger lesions tend to be more heterogeneous due to necrosis or hemorrhage [13]. Intra-lesional echogenic foci due to calcifications can be also observed. At Color Doppler, this lesion appears as a hypervascular mass, which may demonstrate an afferent blood vessel [13]. CEUS reveals a 

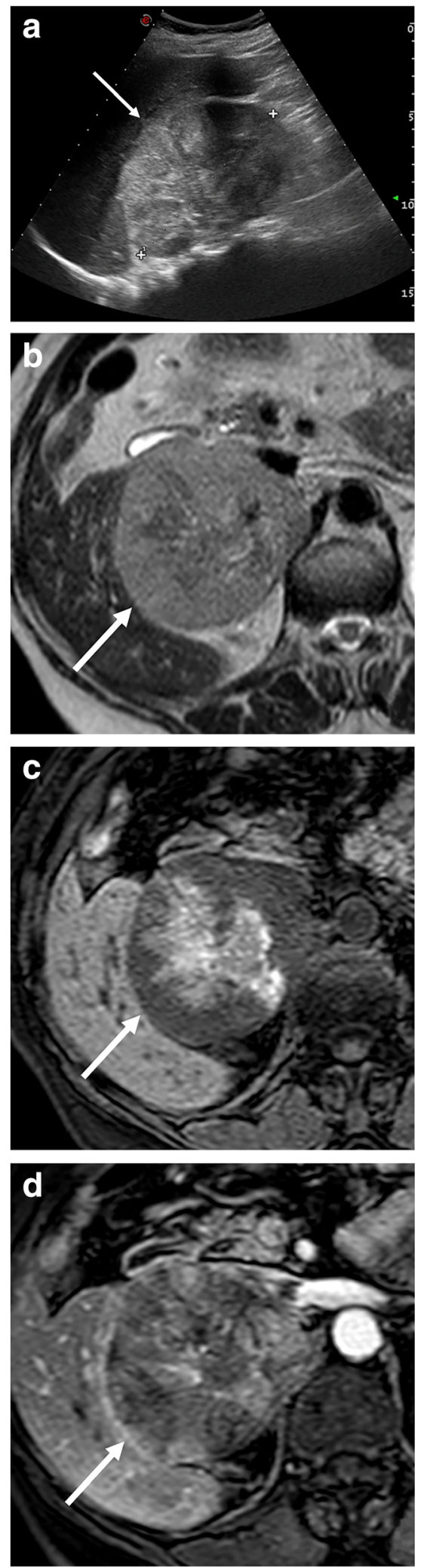

Fig. 8 Right cortical carcinoma in a 70-year-old woman admitted to the emergency department for atraumatic abdominal pain. US (a) shows a large heterogeneous right adrenal mass (arrow). Axial T2weighted spin echo (b), three-dimensional fat-suppressed T1weighted gradient recalled echo images before contrast injection (c), in arterial phase (d) images confirm the presence of a large right adrenal mass with necrotic and hemorrhagic components which present as areas of high signal on unenhanced T1-weighted (c) images and non-enhancing areas after contrast injection (d)

hypervascularized lesion with early enhancement similar to that of pheochromocytoma [14].

On CT, adrenal carcinoma shows a heterogeneous appearance due to necrosis, calcifications, and hemorrhage. After intravenous contrast injection, it demonstrates heterogeneous and mainly peripheral enhancement [66]. As previously reported, the RPW of the carcinoma tends to be less than 40\% [67]. Invasion of adjacent structures such as kidney, inferior vena cava, and splenic vessels are common as well as liver metastasis and retroperitoneal lymph nodal locations.

On MR, cortical carcinoma shows low signal intensity on T1-weighted images, high signal on T2-weighted images, and strong and heterogeneous contrast enhancement with slow washout $[68,69]$. Hemorrhagic areas may present as intra-lesional areas of high signal intensity on T1-weighted images (Fig. 8) [69]. Rarely, focal loss of signal intensity on out-of-phase images due to foci of intracytoplasmic fat may be observed [69].

Despite ${ }^{18}$ F-FDG-PET/CT not completely included in the management algorithms of adrenal malignancies, the potential value of this method has been proposed as a second-line test in ruling out suspected recurrences found by CT [70]. Furthermore, a recent study by Cistaro et al. focused on the comparison of ${ }^{18} \mathrm{~F}$-FDG-PET/CT with conventional imaging, showing better accuracy of ${ }^{18} \mathrm{~F}$-FDG-PET/CT (93.4\%) in comparison to CECT (75\%) [71]. Nevertheless, further studies will be necessary to confirm these results and its role in the clinical setting.

\section{Lymphoma}

The primary adrenal lymphoma is very rare, with the secondary adrenal involvement being more frequent [72]. In $50 \%$ of cases, a bilateral location is observed, generally associated with retroperitoneal pathologic lymph nodes [72]. On US, lymphoma appears as hypoechoic with a homogenous or heterogeneous appearance [73]. On CT, primary adrenal lymphoma appears as a single complex hypoattenuating mass, without intra-lesional calcifications, showing slight contrast enhancement (Fig. 9) [74]. In secondary 

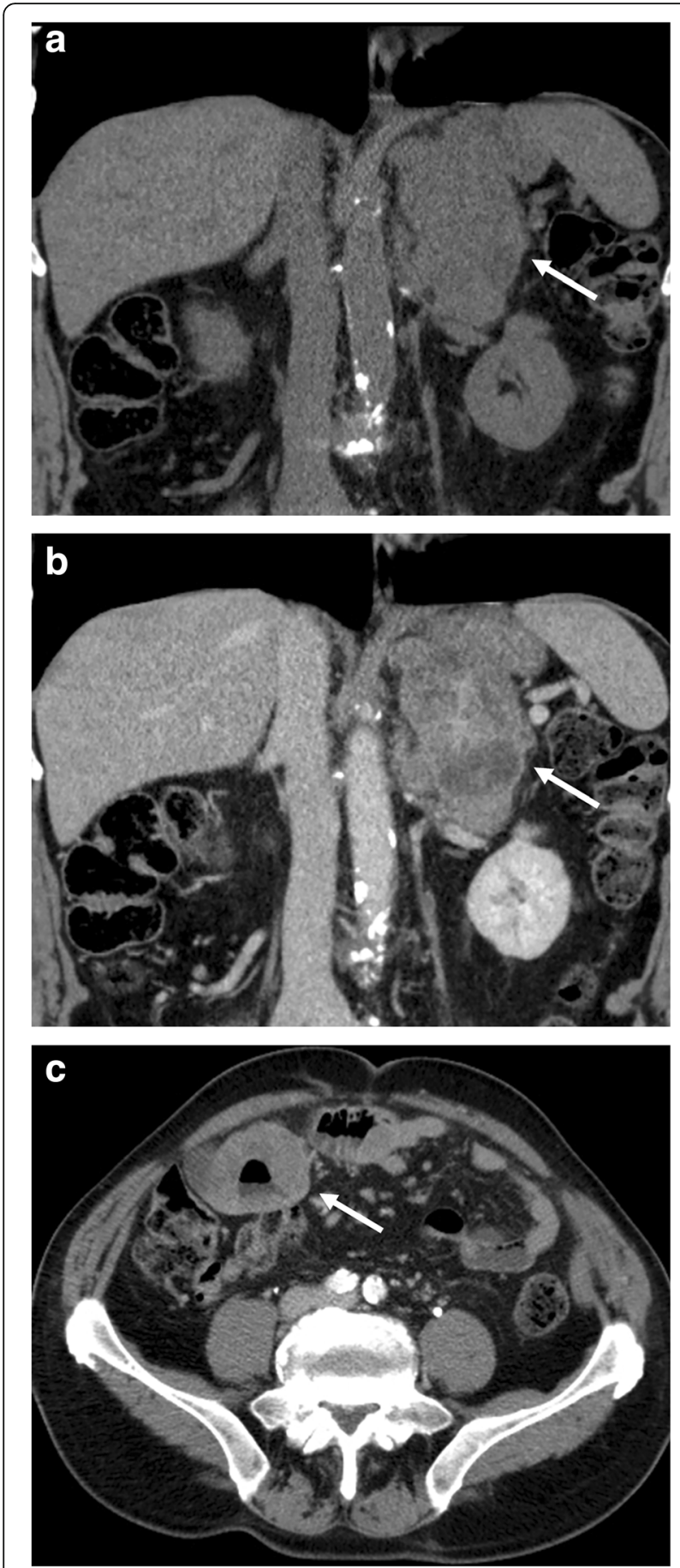

Fig. 9 Left adrenal lymphomatous lesion in a 70-year-old man with non-Hodgkin lymphoma. Coronal reformatted CT images before (a) and after contrast injection in portal phase (b) show a large left adrenal mass (arrow) with inhomogeneous enhancement. Axial CT image of the lower abdomen in portal phase ( $\mathbf{c}$; arrow) shows the gastrointestinal involvement by lymphoma as aneurysmal dilatation of some loops of the small bowel adrenal lymphoma, the adrenals appear enlarged but maintain normal morphology also being more homogeneous than primary lesions. On CECT, secondary lymphomas show slight contrast enhancement and slow washout [72]. At MR, adrenal lymphoma is hypo/isointense on T1-weighted and slightly hyperintense on T2-weighted images [72]. On DWI, lymphomatous lesions always show a restricted pattern of diffusion due to their hypercellularity and high nuclear-to-cytoplasmic ratio, leading to decreased diffusivity of water molecules [75-78]. Nevertheless, this imaging feature is not specific for adrenal lymphoma, but detectable in all locations [79].

\section{Metastases}

Metastases are the most frequent malignant adrenal lesions. The most common primary tumor metastasizing to the adrenal glands is lung cancer followed by breast, colon, melanoma, kidney, and hepatocellular carcinoma [80]. Bilateral metastases are more common than unilateral metastasis [80]. Metastases are clinically silent, but in some cases extensive involvement of both glands can determine adrenal insufficiency. CT is the method of choice in oncologic patients, while US is used predominantly in pediatric patients. MR is a second-instance method, useful only for further characterization of adrenal lesions. ${ }^{18}$ F-FDG-PET/CT can be helpful to differentiate fat poor adenoma from small metastasis [81].

At CT, adrenal metastases appear as focal masses with strong and prolonged enhancement on the portal venous phase, usually more than $120 \mathrm{HU}$, but slower washout than adenomas (Fig. 10) [21]. At MR, imaging features depend on the type of primary tumor, but generally appear hypointense on T1-weighted and moderately hyperintense on T2-weighted images. Metastases from melanoma may show high signal intensity on T1-weighted images. Moreover, adrenal metastases may show hypointense areas on T2-weighted images with no enhancement after contrast injection due to hemorrhagic intra-lesional components (Fig. 11). Another important imaging feature, which may be helpful to differentiate metastases from adenomas, is the lack of signal loss on out-of-phase images [51]. Conversely, the differential diagnosis between metastasis and primary carcinoma is very difficult, as well as in other districts, with carcinoma generally showing higher local invasiveness [82].

As a functional imaging modality, which provides glucose metabolic information on malignant tumors, ${ }^{18} \mathrm{~F}-\mathrm{FDG}$-PET/CT has shown encouraging results for the detection of adrenal metastases. Recently, a meta-analysis of $\mathrm{Wu}$ et al. evaluating 707 lung cancer patients with 810 adrenal masses has reported an 

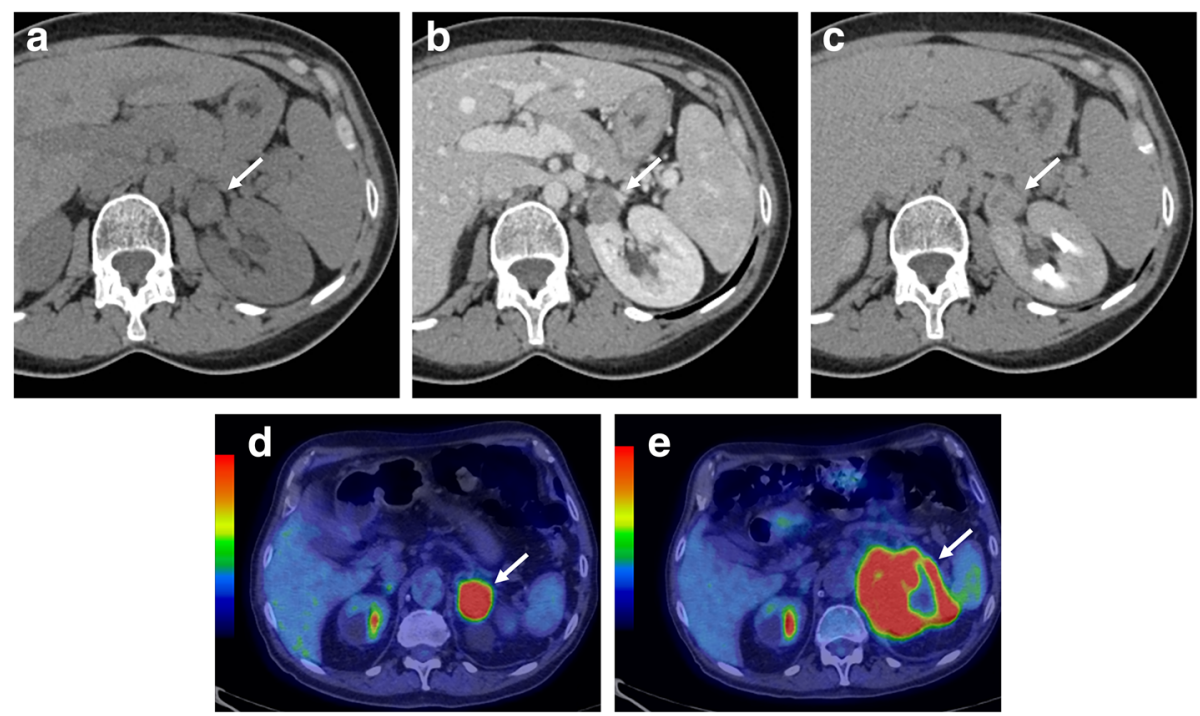

Fig. 10 Left adrenal metastasis in a 62-year-old woman with lung cancer. Unenhanced $C T(\mathbf{a})$, portal (b), and late phase (c) images show a left adrenal mass (arrow) with attenuation value of $26 \mathrm{HU}$ on unenhanced CT (a) and "absolute percentage washout" of 15\%. Staging ${ }^{18} \mathrm{~F}-\mathrm{FDG}$ PET/CT (d) performed 3 months later revealed a left adrenal lesion (arrow) with high metabolic activity (SUV $\max 14 \mathrm{~g} / \mathrm{mL}$ ) and increased in size in comparison with CT images. After chemotherapy, restaging ${ }^{18} \mathrm{~F}-\mathrm{FDG}-\mathrm{PET} / \mathrm{CT}$ (e) showed morpho-functional disease progression of the adrenal lesion (arrow) with signs of central necrosis (SUV $\max 19 \mathrm{~g} / \mathrm{mL}$ with central necrotic area of absence of ${ }^{18} \mathrm{~F}$-FDG uptake). Images of ${ }^{18} \mathrm{~F}-\mathrm{FDG}$-PET/CT provided by database of Nuclear Medicine Service, Fondazione Istituto G. Giglio, Cefalù, Italy

excellent diagnostic performance of ${ }^{18} \mathrm{~F}$-FDG-PET/CT supporting its clinical value in the differentiation of adrenal metastases from benign masses [83]. A retrospective study conducted by Kim JY et al. on 325 patients evaluated a combination of morpho-functional parameters using $\mathrm{PET} / \mathrm{CT}$ demonstrating that unenhanced attenuation of $>10 \mathrm{HU}$ at $\mathrm{CT}$ imaging and a $\mathrm{SUV}_{\text {max }}$ ratio of $>2.5$ in PET were significantly associated with adrenal metastases [84]. Further, several studies have compared CT with ${ }^{8}$ F-FDG-PET/CT in this setting. The sensitivity of PET remains still lower than $\mathrm{CT}$ due to a limited spatial resolution. For this reason, given a superior specificity, ${ }^{18} \mathrm{~F}$-FDG-PET/CT could be considered as a second-stage imaging study for evaluation of indeterminate adrenal lesions [85].

\section{Neuroblastoma}

Neuroblastoma is the second most common neoplasm in childhood after Wilms tumor, being very rare in adults [86]. This lesion usually presents calcification, necrosis, and intra-lesional hemorrhage [86]. Generally, it is totally asymptomatic, becoming clinically evident when it invades or compresses adjacent structures, metastasizes, or determines a paraneoplastic clinical picture [87].

US is the first imaging modality for this lesion in pediatric patients. Neuroblastoma appears as a large lesion with inhomogeneous echotexture, intra-lesional vascularity at color Doppler, and calcifications in some cases [87]. On CT, it is a lobulated mass, with amorphous or mottled calcifications, usually without capsule and with mild contrast enhancement. Intra-lesional calcifications are detected in $80-90 \%$ of cases and represent an important finding to differentiate this neoplasm from Wilms tumor [86, 87].

MR has shown to be more accurate than CT in the characterization of these tumors. Neuroblastoma usually shows a variable appearance, with low signal on T1-weighted and high signal intensity on T2-weighted images, demonstrating heterogeneous contrast enhancement $[86,87]$.

The MIBG scintigraphy for the detection of this neoplasm has shown equal sensitivity to that for pheochromocytoma [88].

\section{Conclusion}

The widespread use of imaging has dramatically increased the detection of incidental adrenal lesions, which are mostly benign and non-functioning adenomas. Nevertheless, differentiating a benign adrenal lesion from a malignant one can be challenging and is critical especially in oncology patients, since it will greatly affect patient management. In this setting, imaging plays a crucial role with several tools, which can be employed in the attempt to characterize adrenal lesions. Radiologists should recall the strengths 

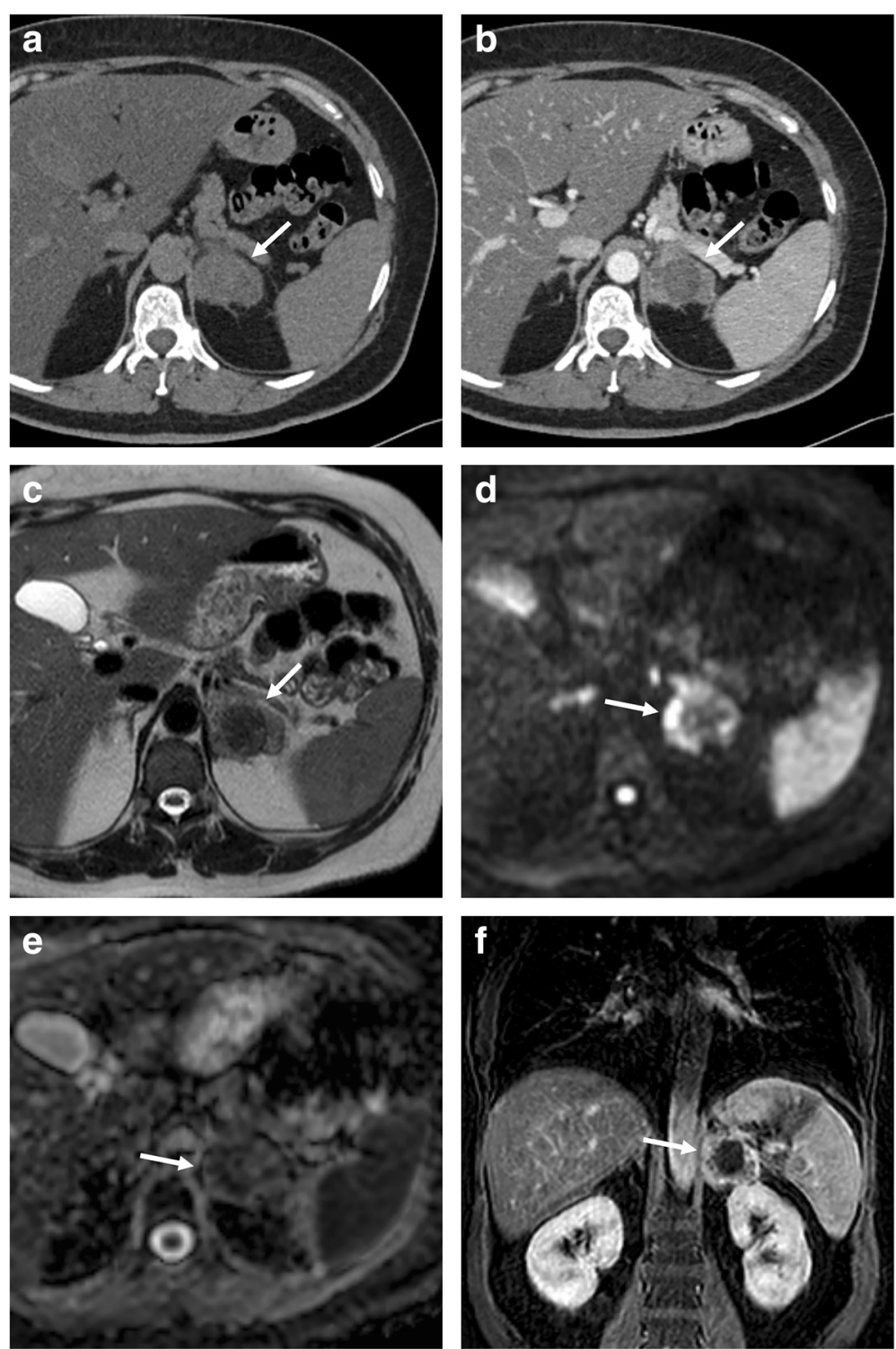

Fig. 11 Left adrenal metastasis in a 58-year-old woman with breast cancer. Axial unenhanced CT (a), portal phase (b), T2-weighted fast spin echo (c), b800 s/mm² diffusion-weighted image (d), ADC map (e), and coronal three-dimensional fat-suppressed T1-weighted gradient recalled echo image in portal phase after contrast injection (f) show a left adrenal mass (arrow). This lesion intra-lesional hemorrhage presenting as

spontaneously hyperdense area on unenhanced CT (a), hypointense on T2-weighted (c), and no enhancement on CT (b) and MR (f) images after contrast injection. The peripheral solid component of the lesion shows also restricted pattern of diffusion (d, e)

and weaknesses of imaging modalities to better help clinicians and surgeons in the management of patients, avoiding a misinterpretation of imaging features which frequently overlap between benign and malignant conditions.

\section{Funding}

The authors state that this article did not receive any funding.

\section{Authors' contributions}

Study concepts and design: DA, FA, MM, MG; literature research: FM, GP, PT surgical cases provided by: GDB, AG; figures saved and prepared by: $A B$ and
PA; manuscript preparation: DA, FA, PA, LMS, MG; manuscript editing: SP, LLG, MM; all authors read and approved the final manuscript.

\section{Competing interests}

The authors declare that they have no competing interests.

\section{Publisher's Note}

Springer Nature remains neutral with regard to jurisdictional claims in published maps and institutional affiliations.

\section{Author details}

${ }^{1}$ Unità di Radiologia Diagnostica ed Interventistica, IRCCS Istituto Ortopedico Galeazzi, Via Riccardo Galeazzi 4, 20161 Milan, Italy. ${ }^{2}$ Dipartimento di 
Biomedicina, Neuroscienze e Diagnostica Avanzata, Università degli studi di Palermo, Via del Vespro 127, 90127 Palermo, Italy. ${ }^{3}$ Department of Radiological Sciences, Nuclear Medicine Service, Fondazione Istituto G. Giglio, Contrada Pietrapollastra-Pisciotto, 90015 Cefalu, Italy. ${ }^{4}$ Department of General Surgery, Urgency and Organ Transplantation, University of Palermo, Via del Vespro 127, 90127 Palermo, Italy. ${ }^{5}$ Department of Biomedical Sciences for Health, Università degli Studi di Milano, Via Festa del Perdono 7, 20122 Milan, Italy. ${ }^{6}$ Department PROMISE, University of Palermo, Via del Vespro 127, 90127 Palermo, Italy.

\section{Received: 10 September 2018 Accepted: 3 January 2019}

\section{Published online: 25 January 2019}

\section{References}

1. Galia M, Albano D, Narese D et al (2016) Whole body MRI in patients with lymphoma: collateral findings. Radiol Med. https://doi.org/10.1007/s11547016-0658-x

2. Sconfienza LM, Mauri G, Muzzupappa C et al (2015) Relevant incidental findings at abdominal multi-detector contrast-enhanced computed tomography: a collateral screening? World J Radiol. https://doi.org/10.4329/ wjr.v7.i10.350

3. Mayo-Smith WW, Song JH, Boland GL, et al (2017) Management of incidental adrenal masses: a White Paper of the ACR Incidental Findings Committee. J Am Coll Radiol. https://doi.org/10.1016/j.jacr.2017.05.001

4. Kebebew $E$ (2017) Management of adrenal masses in children and adults. Springer. https://doi.org/10.1007/978-3-319-44136-8

5. Johnson PT, Horton KM, Fishman EK (2009) Adrenal mass imaging with multidetector CT: pathologic conditions, pearls, and pitfalls. Radiographics. https://doi.org/10.1148/rg.295095027

6. Adam SZ, Nikolaidis P, Horowitz JM et al (2016) Chemical shift MR imaging of the adrenal gland: principles, pitfalls, and applications. Radiographics. https://doi.org/10.1148/rg.2016150139

7. Chong S, Lee KS, Kim HY (2006) Integrated PET-CT for the characterization of adrenal gland lesions in cancer patients: diagnostic efficacy and interpretation pitfalls. Radiographics. https://doi.org/10.1148/rg.266065057

8. Kim S, Cho J (2017) Oncologic imaging: urology. Springer. https://doi.org/10. 1007/978-3-662-45218-9

9. Cerny JC (1977) Anatomy of the adrenal gland. Urol Clin North Am Jun 4(2): 169-177

10. Elsayes KM, Mukundan G, Narra VR et al (2004) Adrenal masses: MR imaging features with pathologic correlation. Radiographics. https://doi.org/10.1148/ rg.24si045514

11. Blake MA, Holalkere NS, Boland GW (2008) Imaging techniques for adrenal lesion characterization. Radiol Clin N Am. https://doi.org/10.1016/j.rcl.2008. 01.003

12. Schirpenbach C, Reincke M (2007) Primary aldosteronism: current knowledge and controversies in Conn's syndrome. Nat Clin Pract Endocrinol Metab. https://doi.org/10.1038/ncpendmet0430

13. Fan J, Tang J, Fang J et al (2014) Ultrasound imaging in the diagnosis of benign and suspicious adrenal lesions. Med Sci Monit. https://doi.org/10. 12659/MSM.890800

14. Friedrich-Rust M, Glasemann T, Polta A et al (2011) Differentiation between benign and malignant adrenal mass using contrast-enhanced ultrasound. Ultraschall Med. https://doi.org/10.1055/s-0031-1273408

15. Caoili E, Korobkin M, Francis IR et al (2002) Adrenal masses: characterization with combined unenhanced and delayed enhanced CT. Radiology. https:// doi.org/10.1148/radiol.2223010766

16. Boland GW, Blake MA, Hahn PF, Mayo-Smith WW (2008) Incidental adrenal lesions: principles, techniques, and algorithms for imaging characterization. Radiology. https://doi.org/10.1148/radiol.2493070976

17. Blake MA, Kalra MK, Sweeney AT et al (2006) Distinguishing benign from malignant adrenal masses: multi-detector row CT protocol with 10-minute delay. Radiology. https://doi.org/10.1148/radiol.2382041514

18. Peña CS, Boland GW, Hahn PF, Lee MJ, Mueller PR (2000) Characterization of indeterminate (lipid-poor) adrenal masses: use of washout characteristics at contrast-enhanced CT. Radiology. https://doi.org/10.1148/radiology.217.3. ro0dc29798

19. Sangwaiya MJ, Boland GW, Cronin CG, et-al (2010) Incidental adrenal lesions: accuracy of characterization with contrast-enhanced washout multidetector CT-10-minute delayed imaging protocol revisited in a large patient cohort. Radiology DOl:https://doi.org/10.1148/radiol.10091386
20. Varghese JC, Hahn PF, Papanicolaou N, Mayo- Smith WW, Gaa JA, Lee MJ (1997) MR differentiation of phaeochromocytoma from other adrenal lesions based on qualitative analysis of T2 relaxation times. Clin Radiol DOI: https://doi.org/10.1016/S0009-9260(97)80252-8

21. Mayo-Smith WW, Boland GW, Noto RB et al (2001) State-of-the-art adrenal imaging. Radiographics. https://doi.org/10.1148/radiographics.21.4 g01j121995

22. Haider MA, Ghai S, Jhaveri K, Lockwood G (2004) Chemical shift MR imaging of hyperattenuating (> $10 \mathrm{HU}$ ) adrenal masses: does it still have a role? Radiology. https://doi.org/10.1148/radiol.2313030676

23. Ilias I, Pacak K (2004) Current approaches and recommended algorithm for the diagnostic localization of pheochromocytoma. J Clin Endocrinol Metab. https://doi.org/10.1210/jc.2003-031091

24. Schieda N, Siegelman ES (2017) Update on CT and MRI of adrenal nodules. AJR Am J Roentgenol. https://doi.org/10.2214/AJR.16.17758

25. Miller FH, Wang Y, McCarthy RJ et al (2010) Utility of diffusion-weighted MR in characterization of adrenal lesions. AJR Am J Roentgenol. https://doi.org/ 10.2214/AJR.09.2891

26. Sandrasegaran K, Patel AA, Ramaswamy R et al (2011) Characterization of adrenal masses with dif- fusion-weighted imaging. AJR Am J Roentgenol. https://doi.org/10.2214/AJR.10.4583

27. Qin HY, Sun HR, Li YJ, Shen BZ (2012) Application of CT perfusion imaging to the histological differentiation of adrenal gland tumors. Eur J Radiol. https://doi.org/10.1016/j.ejrad.2010.12.072

28. Helck A, Hummel N, Meinel FG, Johnson T, Nikolaou K, Graser A (2014) Can single-phase dual-energy $C T$ reliably identify adrenal adenomas? Eur Radiol. https://doi.org/10.1007/s00330-014-3192-z

29. Ho LM, Paulson EK, Brady MJ, Wong TZ, Schindera ST (2008) Lipid-poor adenoma on unenhanced $\mathrm{CT}$ : does histogram analysis increase sensitivity compared with a mean attenuation threshold? AJR Am J Roentgenol. https://doi.org/10.2214/AJR.07.3150

30. Romeo V, Maurea S, Cuocolo R et al (2018) Characterization of adrenal lesions on unenhanced MRI using texture analysis: a machine-learning approach. J Magn Reson Imaging. https://doi.org/10.1002/jmri.25954

31. Lattin GE Jr, Sturgill ED, Tujo CA et al (2014) From the radiologic pathology archives: adrenal tumors and tumor-like conditions in the adult: radiologicpathologic correlation. Radiographics. https://doi.org/10.1148/rg.343130127

32. Agrons MM, Jensen CT, Habra MA et al (2017) Adrenal cortical hyperplasia: diagnostic workup, subtypes, imaging features and mimics. Br J Radiol. https://doi.org/10.1259/bjr.20170330

33. Park SY, Park BK, Park JJ, Kim CK (2016) Differentiation of adrenal hyperplasia from adenoma by use of $C T$ densitometry and percentage washout. AJR Am J Roentgenol. https://doi.org/10.2214/AJR.15.14558

34. Lumachi F, Zucchetta P, Marzola MC et al (2002) Usefulness of CT scan, MRI and radiocholesterol scintigraphy for adrenal imaging in Cushing's syndrome. Nucl Med Commun. https://doi.org/10.1097/00006231200205000-00007

35. Alshahrani MA, Bin Saeedan M, Alkhunaizan T, Aljohani IM, Azzumeea FM (2018) Bilateral adrenal abnormalities: imaging review of different entities. Abdom Radiol. https://doi.org/10.1007/s00261-018-1670-5

36. Huang YC, Tang YL, Zhang XM, Zeng NL, Li R, Chen TW (2015) Evaluation of primary adrenal insufficiency secondary to tuberculous adrenalitis with computed tomography and magnetic resonance imaging: current status. World J Radiol. https://doi.org/10.4329/wjr.v7.i10.336

37. Rana Al, Kenney PJ, Lockhart ME et al (2004) Adrenal gland hematomas in trauma patients. Radiology. https://doi.org/10.1148/radiol.2303021345

38. Jordan E, Poder L, Courtier J et-al (2012) Imaging of nontraumatic adrenal hemorrhage. AJR Am J Roentgenol. DOl:https://doi.org/10.2214/AJR.11.7973

39. Carvounis E, Marinis A, Arkadopoulos N, Theodosopoulos T, Smyrniotis V (2006) Vascular adrenal cysts: a brief review of the literature. Arch Pathol Lab Med. https://doi.org/10.1043/1543-2165(2006)130[1722:VACABR]2.0.CO;2

40. Sanal HT, Kocaoglu M, Yildirim D et al (2006) Imaging features of benign adrenal cysts. Eur J Radiol. https://doi.org/10.1016/j.ejrad.2006.08.005

41. Zeiger MA, Thompson GB, Duh QY et al (2009) American Association of Clinical Endocrinologists and American Association of Endocrine Surgeons Medical Guidelines for the Management of Adrenal Incidentalomas: executive summary of recommendations. Endocr Pract. https://doi.org/10. 4158/EP.15.5.450

42. Rao P, Kenney PJ, Wagner BJ, Davidson AJ (1997) Imaging and pathologic features of myelolipoma. Radiographics. https://doi.org/10.1148/ radiographics.17.6.9397452 
43. Galia M, Albano D, Bruno A et al (2017) Imaging features of solid renal masses. Br J Radiol. https://doi.org/10.1259/bjr.20170077

44. Sharma MC, Gill SS, Kashyap S, Nabi G, Mishra MC (1998) Adrenal lipoma. A case report. Urol Int. https://doi.org/10.1159/000030265

45. Ghavamian R, Pullman JM, Menon M (1998) Adrenal lipoma: an uncommon presentation of the incidental asymptomatic adrenal mass. Br J Urol. https:// doi.org/10.1046/j.1464-410x.1998.00711.x

46. Blake MA, Kalra MK, Maher MM et al (2004) Pheochromocytoma: an imaging chameleon. Radiographics DOl:https://doi.org/10.1148/rg 24 si045506

47. Leung K, Stamm M, Raja A et al (2013) Pheochromocytoma: the range of appearances on ultrasound, CT, MRI, and functional imaging. AJR Am J Roentgenol DOl:https://doi.org/10.2214/AJR.12.9126

48. Al Bunni F, Deganello A, Sellars ME, Schulte KM, Al-Adnani M, Sidhu PS (2014) Contrast-enhanced ultrasound (CEUS) appearances of an adrenal phaeochromocytoma in a child with Von Hippel-Lindau disease. J Ultrasound. https://doi.org/10.1007/s40477-014-0083-8

49. Patel J, Davenport MS, Cohan RH, Caoili EM (2013) Can established CT attenuation and washout criteria for adrenal adenoma accurately exclude pheochromocytoma? AJR Am J Roentgenol. https://doi.org/10.2214/AJR.12.9620

50. Jacques AE, Sahdev A, Sandrasagara M et al (2008) Adrenal phaeochromocytoma: correlation of MRI appearances with histology and function. Eur Radiol. https://doi.org/10.1007/s00330-008-1073-z

51. Dong Y, Liu Q (2012) Differentiation of malignant from benign pheochromocytomas with diffusion-weighted and dynamic contrastenhanced magnetic resonance at 3.0 T. J Comput Assist Tomogr. https:// doi.org/10.1097/RCT.0b013e31825975f8

52. Kim S, Salibi N, Hardie AD et al (2009) Characterization of adrenal pheochromocytoma using respiratory-triggered proton MR spectroscopy: initial experience. AJR Am J Roentgenol. https://doi.org/10.2214/AJR.07.4027

53. Bombardieri E, Giammarile F, Aktolun C et al (2010) 1311/1231metaiodobenzylguanidine (mIBG) scintigraphy: procedure guidelines for tumour imaging. Eur J Nucl Med Mol Imaging. https://doi.org/10.1007/ s00259-010-1545-7

54. Sharma P, Dhull VS, Arora S et al (2014) Diagnostic accuracy of (68)GaDOTANOC PET/CT imaging in pheochromocytoma. Eur J Nucl Med Mol Imaging. https://doi.org/10.1007/s00259-013-2598-1

55. Amodru V, Guerin C, Delcourt S et al (2018) Quantitative (18)F-DOPA PET/CT in pheochromocytoma: the relationship between tumor secretion and its biochemical phenotype. Eur J Nucl Med Mol Imaging. https://doi.org/10. 1007/s00259-017-3833-y

56. Agrusa A, Romano G, Salamone G et al (2015) Large cavernous hemangioma of the adrenal gland: laparoscopic treatment. Report of a case. Int J Surg Case Rep. https://doi.org/10.1016/j.jiscr.2015.09.040

57. Ellis CL, Banerjee P, Carney E, Sharma R, Netto GJ (2011) Adrenal lymphangioma: clinicopathologic and immunohistochemical characteristics of a rare lesion. Human Pathol. https://doi.org/10.1016/j.humpath.2010.10.023

58. Rowe SP, Bishop JA, Prescott JD, Salvatori R, Fishman EK (2016) CT appearance of adrenal cystic lymphangioma: radiologic-pathologic correlation. AJR Am J Roentgenol. https://doi.org/10.2214/AJR.15.14786

59. Inokuchi $T$, Takiuchi $H$, Moriwaki $Y$ et al (2006) Retroperitoneal ancient schwannoma presenting as an adrenal incidentaloma: CT and MR findings. Magn Reson Imaging. https://doi.org/10.1016/j.mri.2006.07.015

60. Guo YK, Yang ZG, Li Y et al (2007) Uncommon adrenal masses: CT and MR features with histopathologic correlation. Eur J Radiol. https://doi.org/10. 1016/j.ejrad.2006.12.011

61. Maweja S, Materne R, Detrembleur N et al (2007) Adrenal ganglioneuroma. Am J Surg. https://doi.org/10.1016/j.amjsurg.2007.01.034

62. Radin R, David CL, Goldfarb H, Francis IR (1997) Adrenal and extra-adrenal retroperitoneal ganglioneuroma: imaging findings in 13 adults. Radiology. https://doi.org/10.1148/radiology.202.3.9051020

63. Tahar GT, Nejib KN, Sadok SS, Rachid LM (2008) Adrenocortical oncocytoma: a case report and review of literature. J Pediatr Surg. https://doi.org/10. 1016/j.jpedsurg.2007.12.067

64. Bisceglia M, Ludovico O, Di Mattia A et al (2004) Adrenocortical oncocytic tumors: report of 10 cases and review of the literature. Int J Surg Pathol. https://doi.org/10.1177/106689690401200304

65. Shah RK, Oto A, Ozkan OS et al (2004) Adrenal oncocytoma: US and CT findings. JBR-BTR 87:180-182

66. Ng L, Libertino JM (2003) Adrenocortical carcinoma: diagnosis, evaluation and treatment. J Urol. https://doi.org/10.1097/01.ju.0000030148.59051.35
67. Agrusa A, Romano G, Navarra G et al (2017) Innovation in endocrine surgery: robotic versus laparoscopic adrenalectomy. Meta-analysis and systematic literature review. Oncotarget. https:/doi.org/10.18632/oncotarget.22059

68. Bharwani N, Rockall AG, Sahdev A et al (2011) Adrenocortical carcinoma: the range of appearances on CT and MRI. AJR Am J Roentgenol. https://doi.org/ 10.2214/AJR. 10.5540

69. Ferrozzi F, Bova D (1995) CT and MR demonstration of fat within an adrenal cortical carcinoma. Abdom Imaging 20(3):272-274

70. Ardito A, Massaglia C, Pelosi E et al (2015) 18F-FDG PET/CT in the postoperative monitoring of patients with adrenocortical carcinoma. Eur J Endocrinol. https://doi.org/10.1530/EJE-15-070

71. Cistaro A, Niccoli Asabella A, Coppolino P et al (2015) Diagnostic and prognostic value of 18F-FDG PET/CT in comparison with morphological imaging in primary adrenal gland malignancies - a multicenter experience. Hell J Nucl Med. https://doi.org/10.1967/s002449910202

72. Leite NP, Kased N, Hanna RF et al (2007) Cross-sectional imaging of extranodal involvement in abdominopelvic lymphoproliferative malignancies. Radiographics. https://doi.org/10.1148/rg.276065170

73. Kim H, Kim KW, Park MS, Kim H (2008) Lymphoma presenting as an echogenic periportal mass: sonographic findings. J Clin Ultrasound. https:// doi.org/10.1002/jcu.20453

74. Rashidi A, Fisher SI (2013) Primary adrenal lymphoma: a systematic review. Ann Hematol. https://doi.org/10.1007/s00277-013-1812-3

75. Albano D, Patti C, Matranga D, Lagalla R, Midiri M, Galia M (2018) Wholebody diffusion-weighted MR and FDG-PET/CT in Hodgkin lymphoma: predictive role before treatment and early assessment after two courses of ABVD. Eur J Radiol. https://doi.org/10.1016/j.ejrad.2018.04.014

76. Albano D, Patti C, Lagalla R, Midiri M, Galia M (2017) Whole-body MRI, FDG$\mathrm{PET} / \mathrm{CT}$, and bone marrow biopsy, for the assessment of bone marrow involvement in patients with newly diagnosed lymphoma. J Magn Reson Imaging. https://doi.org/10.1002/jmri.25439

77. Galia M, Albano D, Tarella C et al (2018) Whole body magnetic resonance in indolent lymphomas under watchful waiting: the time is now. Eur Radiol. https://doi.org/10.1007/s00330-017-5071-x

78. Albano D, Patti C, La Grutta L, et al (2016) Comparison between wholebody MRI with diffusion-weighted imaging and PET/CT in staging newly diagnosed FDG-avid lymphomas. Eur J Radiol. https://doi.org/10.1016/j.ejrad. 2015.12.006

79. Albano D, La Grutta L, Grassedonio E, et al (2016) Pitfalls in whole body MRI with diffusion weighted imaging performed on patients with lymphoma: what radiologists should know. Magn Reson Imaging. https://doi.org/10. 1016/j.mri.2016.04.023

80. Lam KY, Lo CY (2002) Metastatic tumours of the adrenal glands: a 30-year experience in a teaching hospital. Clin Endocrinol. https://doi.org/10.1046/j. 0300-0664.2001.01435.x

81. Sung YM, Lee KS, Kim BT et al (2008) (18)F-FDG PET versus (18)F-FDG PET/CT for adrenal gland lesion characterization: a comparison of diagnostic efficacy in lung cancer patients. Korean J Radiol. https://doi.org/10.3348/kjr.2008.9.1.19

82. Galia M, Albano D, Picone D et al (2018) Imaging features of pancreatic metastases: a comparison with pancreatic ductal adenocarcinoma. Clin Imaging. https://doi.org/10.1016/j.clinimag.2018.01.016

83. Wu Q, Luo W, Zhao Y, Xu F, Zhou Q (2017) The utility of 18F-FDG PET/CT for the diagnosis of adrenal metastasis in lung cancer: a PRISMA-compliant metaanalysis. Nucl Med Commun. https://doi.org/10.1097/MNM.0000000000000757

84. Kim JY, Kim SH, Lee HJ et al (2013) Utilisation of combined 18F-FDG PET/CT scan for differential diagnosis between benign and malignant adrenal enlargement. Br J Radiol. https://doi.org/10.1259/bjr.20130190

85. Delivanis DA, Bancos I, Atwell TD et al (2018) Diagnostic performance of unenhanced computed tomography and 18 F-fluorodeoxyglucose positron emission tomography in indeterminate adrenal tumours. Clin Endocrinol (Oxf). https://doi.org/10.1111/cen.13448

86. Rha SE, Byun JY, Jung SE, Chun HJ, Lee HG, Lee JM (2003) Neurogenic tumours in the abdomen: tumour types and imaging characteristics. Radiographics. https://doi.org/10.1148/rg.231025050

87. Lonergan GJ, Schwab CM, Suarez ES et al (2002) Neuroblastoma, ganglioneuroblastoma, and ganglioneuroma: radiologic-pathologic correlation. Radiographics. https://doi.org/10.1148/radiographics.22.4.g02j115911

88. Pfluger T, Schmied C, Porn $U$ et al (2003) Integrated imaging using MRI and 1231 metaiodobenzylgua- nidine scintigraphy to improve sensitivity and specificity in the diagnosis of pediatric neuroblastoma. AJR Am J Roentgenol. https://doi.org/10.2214/ajr.181.4.1811115 